\title{
Invited commentary: Anatomy of risk
}

\author{
Femi Oyebode
}

The theoretical notion of risk is sweeping all before it. Politicians, government planners, scientists and doctors, including psychiatrists, are having to confront a more formalised concept of risk. This is not to say that our awareness, as human beings, of notions of risk has hitherto been largely negligible. However, there is an evident contemporary preoccupation with risk, its assessment and management, which signals society's unwillingness to tolerate or bear anything but the most minimal possibility of adverse outcomes. There are competing definitions and concepts of risk which complicate discussions between experts and the lay public. For example, Thompson (1990) distinguishes between 'real' risk, 'observed' risk, and 'perceived' risk. Real risk is thought to reflect how things stand in the real world. That is, it is a term which refers to actual risk, the actual negative consequences which exist. be they random or otherwise. Whereas observed risk is our evaluation of negative consequences based upon a theoretical model of the physical world. This is further distinguished from perceived risk which is conceived of as being the estimate of real risk in the absence of a theoretical model of the world. The distinction which is being drawn is a subtle one but it is an attempt to capture the idea that our perception of risk is judgmental in nature and does not necessarily correspond with objective reality. The underlying assumption, that our subjective evaluation of risk may not coincide with the true nature of things, is one that psychiatrists are familiar with. The lay perception of the risk that psychiatric patients pose to the public is, at the least, an exaggeration of the objective facts. However, the observed risk, that is the risk calculated by experts that psychiatric patients pose to the public may not be an accurate reflection of the actual risk posed by psychiatric patients.

The search for "complete certainty in matters of risk and human safety is, both in principle and in practice, fundamentally unachievable" (Valverde, 1991). Nonetheless, the aim is to motivate and further our capacity to predict and control the world we live in. Thus, risk analysis (assessment) can be construed as a formal, structured process that has as its goal the accurate prediction of possible events which, should they transpire, could pose a potential threat to human health and safety. There is a view that an objective, probabilistic approach to the question of risk assessment is preferable to a more subjective approach. In other words, there is a wish to value data which emanate from scientific research over and above the uninformed judgments of a reasonable person. However, we are all of us aware of the disquietude with which the lay public views expert pronouncements on such matters as the BSE crisis and genetically modulated foods among other things. Thus, there is an apparent tension between the wish for a natural scientist's analysis of risk to be the authoritative basis for regulatory decisions and the public's wariness of authority and expertise.

Formally, risk assessment is defined by the Research Council of the American National Academy of Science as "the characterization of potential adverse health effects of human exposures to environmental hazards" and risk management is "the process of evaluating alternative regulatory actions and selecting among them" (National Research Council, 1983). It is recognised that the selection of the appropriate regulatory action requires the use of value judgments on such issues as the acceptability of risk and the reasonableness of the cost of control. What is it then that makes risk acceptable? It is clear that risk is a relative concept. Factors which contribute to the acceptability of risk include the certainty and severity of the risk; the reversibility of the adverse effect; the knowledge and/or familiarity of the risk; whether the risk is voluntarily accepted or involuntarily imposed; whether individuals are compensated for their exposure to the risk; the advantages of the activity under consideration; and the risks and advantages for any alternatives. What is important is that neither risk analysis nor risk management is static in nature. Advances in knowledge or increases in information may radically alter our stance in relation to a potential source of risk.

Davies (1999, this issue) is right to draw our attention to the management of service risk in mental health services, and particularly in focusing on the whole organisation such that clinical risk becomes only a component part of the risk which a service faces. Davies' contribution is that he emphasises the need for risk to be managed at a locality level. His approach is a derivative of a systems approach to risk 
management. It is important to place the contemporary systems approaches to risk management in the context of the development of a study of human factors in failures of systems. Much of the original theory in this area of work occurred in the setting of man-machine interface with the fundamental goal being the improvement of the design of dials, control knobs and panel layouts. With the shift from classical to systems ergonomics, there was a recognition that people and the machines they operated constituted a single unified system. Error studies therefore concentrated as much on human error in man-machine systems as on the contribution of structural design faults to error causation. There is a further conceptual leap which is required in applying systems approaches to health services. Man-made systems in psychiatry are composed, for the most part, of abstract structures and processes. Therefore, the interaction with these structures and processes is less visible to analysis. Where dials and knobs are readily altered, assessment procedures in psychiatry which are by definition embedded in culture are far more recalcitrant to study and change. Furthermore, the design faults of such abstract entities are more difficult to apprehend.

Davies proposes an analysis of risk which depends on the component parts of a service. There is at least one other mode of scrutinising risk. This would be to concentrate on functions, perhaps in relation to particular activities such as the recognition and treatment of depressive disorders. Then a flow chart of patient interactions with the health service could be developed and the critical interfaces identified for potential risk. Next, a risk management regulatory programme could be explicitly formulated. The advantage of this approach is that it deconstructs the complexity of clinical work into recognisable bits, very much like describing the discrete processes involved in the manufacture of a particular car, in a given factory. The critical nodes and the factors which are liable to corrupt the integrity of these nodes can then be systematically evaluated for fallibility.

The other approach to risk assessment and management derives from the investigation of catastrophic failures. These investigations are also founded on the assumption that human systems are unified. What is clear from these investigations of catastrophic fallures, principally from the aircraft industry, is that accidents are rarely the result of single factors. A classical example is described by Rolfe (1977): An investigation of a plane crash obviously resulting from an obstruction in a fuel supply line showed that pilot error, in attempting to turn back to the aerodrome caused the plane to further lose speed and to stall. This action contravened established procedures. The pilot's safety harness was also faulty and contributed to the fatality of the accident. This case illustrates the complexity of the task of investigating what are clearly catastrophic failures. The homicide inquiries in mental health services are a misconception of the nature of investigations into catastrophic failures. Homicide is not, by definition, evidence of failure. That is, homicide committed by a psychiatric patient is not prima facie evidence of failure. The error is that most inquiries act as if their task is to understand the factors which have contributed to failure, rather than to address the more mundane task of understanding how the homicide came to occur and then to evaluate whether there is any evidence of systems failure. The most that can be said is that homicide, like suicide, is a sentinel event; that is, that it is an event which may, on investigation, reveal systematic errors in man-made systems.

Finally, there is much evidence that risk aversion promotes conservatism. Here, conservatism is defined as a preference for erring on the side of overstating as opposed to understating risk under conditions of uncertainty (Perhac. 1996). This suggests that in the current cultural climate conservatism with respect to safety is on the ascent. There is also evidence that where there is theoretical or model uncertainty for explicating the nature or probability of risk in a given domain as there is with mental health, the administrative answer is to assign an expert committee the entire responsibility for resolving the impact of uncertainties arising from alternative interpretations of possible decisions. The corollary of this is that there is no objecttve procedure to be followed, only negotiated conclusions. This is already evident in the deliberations leading to the National Service Framework and also to the review of mental health legislation.

\section{References}

DAviEs, M. (1999) A simple approach to the management of service risk in a local mental health service. Psychiatric Bulletin. 23, 649-651.

NATIONAL RESEARCH COUNCL (1983) Risk Assessment in the Federal Government: Managing the Process. Washington. DC: National Research Councll.

PERHAC. R. M. (1996) Does risk aversion make a case for conservatism? www. fplc.edu/risk/vol7/fall/perhac.htm

ROLFE, J. M. (1977) Ergonomics and air safety. In Human Aspects of Man-Made Systems (eds S. C. Brown \& J. N. T. Martin), pp. 173-187, Open Untversity Press.

Thompson, P. B. (1990) Risk objectivism and risk subjectivism: when are risks real? www.fplc.edu/risk/ voll/winter/thompson.htm

VALVERDE, L. J. (1991) The cognitive status of risk: a response to Thompson. www.fplc.edu/risk/vol.2/fall/ valuerde.htm

Femi Oyebode, Professor of Psychiatry, Queen Elizabeth Psychiatric Hospital, Mindelsohn Way. Edgbaston, Birmingham B15 2QZ 